\title{
Adaptive Optics Applications in Vision Science
}

\author{
S. S. Olivier
}

This article was submitted to Society of Photo-Optical Instrumentation Engineers West LASE 2003, San Jose, CA, January 25-30, 2003

\section{March 17, 2003}

U.S. Department of Energy

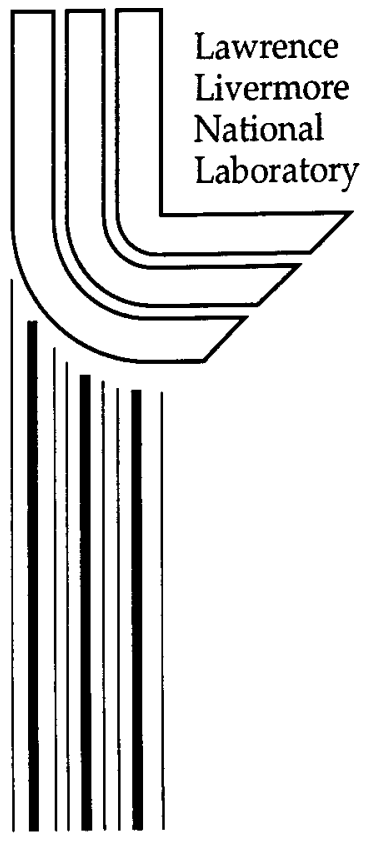




\section{DISCLAIMER}

This document was prepared as an account of work sponsored by an agency of the United States Government. Neither the United States Government nor the University of California nor any of their employees, makes any warranty, express or implied, or assumes any legal liability or responsibility for the accuracy, completeness, or usefulness of any information, apparatus, product, or process disclosed, or represents that its use would not infringe privately owned rights. Reference herein to any specific commercial product, process, or service by trade name, trademark, manufacturer, or otherwise, does not necessarily constitute or imply its endorsement, recommendation, or favoring by the United States Government or the University of California. The views and opinions of authors expressed herein do not necessarily state or reflect those of the United States Government or the University of California, and shall not be used for advertising or product endorsement purposes.

This is a preprint of a paper intended for publication in a journal or proceedings. Since changes may be made before publication, this preprint is made available with the understanding that it will not be cited or reproduced without the permission of the author.

This work was performed under the auspices of the United States Department of Energy by the University of California, Lawrence Livermore National Laboratory under contract No. W-7405-Eng-48.

This report has been reproduced directly from the best available copy.

Available electronically at http://www.doc.gov/bridge

Available for a processing fee to U.S. Department of Energy

And its contractors in paper from

U.S. Department of Energy

Office of Scientific and Technical Information

P.O. Box 62

Oak Ridge, TN 37831-0062

Telephone: (865) 576-8401

Facsimile: (865) 576-5728

E-mail: reports@adonis.osti.gov

Available for the sale to the public from

U.S. Department of Commerce

National Technical Information Service

5285 Port Royal Road

Springfield, VA 22161

Telephone: (800) 553-6847

Facsimile: (703) 605-6900

E-mail: orders@ntis.fedworld.gov

Online ordering: http://www.ntis.gov/ordering.htm

\section{OR}

Lawrence Livermore National Laboratory

Technical Information Department's Digital Library

http://www.llnl.gov/tid/Library.html 


\title{
Adaptive Optics Applications in Vision Science
}

\author{
Scot S. Olivier ${ }^{*}$ \\ Lawrence Livermore National Laboratory, P.O. Box 808, L-395, Livermore, CA 94551
}

\begin{abstract}
Adaptive optics can be used to correct the aberrations in the human eye caused by imperfections in the cornea and the lens and thereby, improve image quality both looking into and out of the eye. Under the auspices of the NSF Center for Adaptive Optics and the DOE Biomedical Engineering Program, Lawrence Livermore National Laboratory has joined together with leading vision science researchers around the country to develop and test new ophthalmic imaging systems using novel wavefront corrector technologies. Results of preliminary comparative evaluations of these technologies in initial system tests show promise for future clinical utility.
\end{abstract}

Keywords: adaptive optics, vision, ophthalmology, wavefront control, MEMS, deformable mirror, liquid crystal, spatial light modulator

\section{INTRODUCTION}

\subsection{Adaptive Optics at Lawrence Livermore National Laboratory}

Adaptive optics have been a key enabling technology for projects at Lawrence Livermore National Laboratory (LLNL) in laser beam control and imaging. Historically, adaptive optics have played an important role in the operation of high power laser systems at LLNL over the past three decades. Currently, the next giant laser system being developed at LLNL, the National Ignition Facility, is designed to be operated with adaptive optics on each of the 192 separate beam lines [1]. These adaptive optics will enable accurate focusing of light into the target chamber for nuclear physics experiments.

Beginning in 1990, based on both adaptive optics and laser expertise developed for high power laser systems, LLNL pioneered the development of adaptive optics for large astronomical telescopes utilizing a sodium-layer laser guide star to significantly improve the observable fraction of the sky. The first sodium-layer laser guide star adaptive optics system, built by LLNL for the 3-meter Shane Telescope at the University of California's Lick Observatory, became operational in 1996 [2]. Currently, this system remains the only such facility in operation, world-wide [3].

Based on the work at Lick Observatory, LLNL also played a key role in the development of the first adaptive optics and sodium-layer laser guide star systems for the Keck Observatory. The adaptive optics system for the 10-meter Keck II Telescope became operational in 1999 [4], and the Keck sodium-layer laser guide star system was deployed and first tested on the telescope in 2001. Final integration of the Keck laser guide star and adaptive optics systems is ongoing [5].

\subsection{National Science Foundation Center for Adaptive Optics}

Beginning in 1998, based on the forefront work in the development of astronomical sodium-layer laser guide star adaptive optics facilities at Lick and Keck Observatories, LLNL entered into a partnership with the University of California, Santa Cruz (UCSC) along with a large number of other institutions, to propose the formation of the NSF Science and Technology Center for Adaptive Optics (CfAO). The CfAO, which received its initial funding in November, 1999, seeks to advance the state of the art of adaptive optics in the fields of astronomy and vision science. Headquartered at UCSC, this multiinstitutional Center has 11 major nodes at academic institutions, 5 at National Laboratories, and 11 currently active industrial partnerships. Further industry partnerships are encouraged, and an Industrial Affiliates program is being initiated in 2003. Additional information can be found on the $\mathrm{CfAO}$ website at www.ucolick.org/ cfao.

\footnotetext{
*Correspondence: email: olivierl@IInl.gov; phone: 925-423-6483; fax: 925-422-1796
} 
Since its inception, the CfAO has undertaken a vigorous research and development program in advanced adaptive optics technologies [6]. CfAO research activities include development and characterization of micro-electro-mechanical systems (MEMS) deformable mirror (DM) technology [7], as well as development and characterization of high-resolution adaptive optics systems using liquid crystal (LC) spatial light modulator (SLM) technology [8].

\subsection{CfAO Vision Science Program}

The vision science work within the CfAO is predicated on the pioneering research carried out at the University of Rochester to apply adaptive optics to the correction of the wave aberration of the human eye [9]. Figure 1 illustrates schematically how adaptive optics can be used to compensate for the wave aberration of the eye.

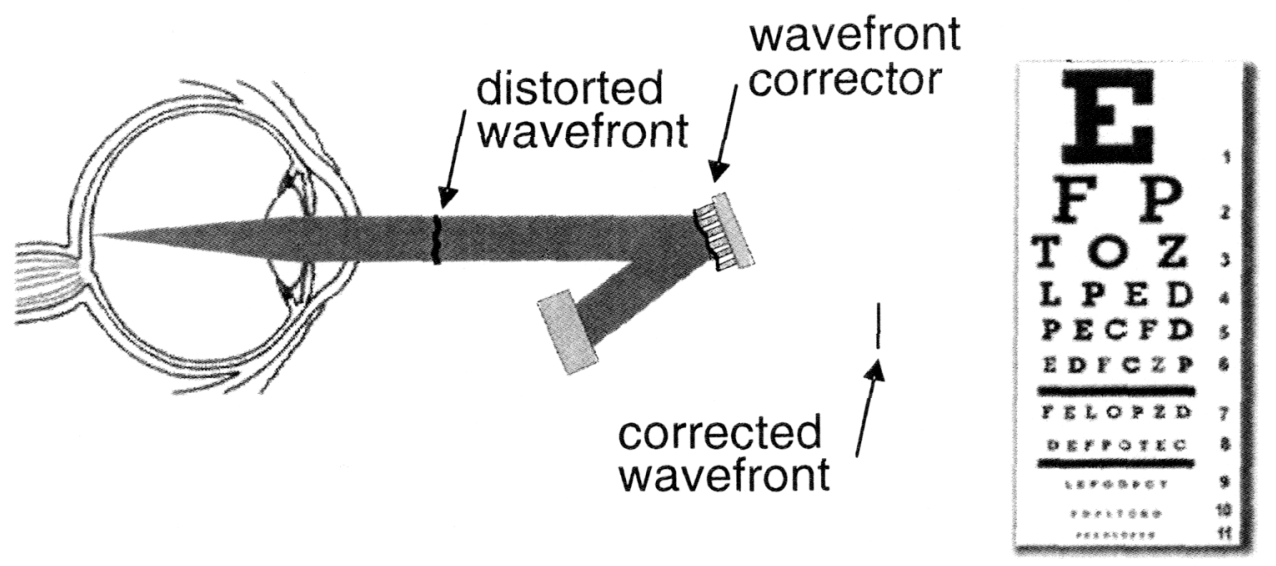

Figure 1 - An adaptive optics system can be used to correct the aberrations in a subject's eye and allow detailed studies of visual performance under a variety of conditions.

A long-term goal of the CfAO vision science program is to commercialize compact AO systems for ophthalmic applications. Two classes of ophthalmic instruments are currently under development within the CfAO. The first class of instruments includes those used for retinal imaging. In simple terms, adaptive optics can be incorporated into fundus cameras to provide ophthalmologists with high-resolution retinal imaging for diagnostic and surgical applications. The second class of instruments includes those used to prescribe vision correction technologies. Once again, in simple terms, adaptive optics can be used to replace the phoropter in order to allow optometrists to assess high-order aberrations in the eye while the patient directly observes the visual benefit of correction. Permanent correction of high-order aberrations would then be accomplished with advanced vision correction techniques, such as custom laser eye surgery or custom contact lenses. By incorporating new compact wavefront corrector technologies, such as MEMS DM or LC SLM devices, along with the current generation of commercial wavefront sensing technology, it should be possible to extend the functionality of the fundus camera and phoropter to higher resolution, while maintaining a clinically viable instrument profile.

\section{LIQUID CRYSTAL SPATIAL LIGHT MODULATOR VISION SYSTEM}

\subsection{LC SLM Technology}

A high-resolution liquid crystal spatial light modulator is commercially available from Hamamatsu, and has been previously used to demonstrate high-order phase compensation in a laboratory test-bed [8]. The advantages of this technology include compactness $(2 \mathrm{~cm}$ clear aperture), low cost $(\sim \$ 30 \mathrm{k})$, ease of use (driven from standard VGA display card), and high resolution correction ( $480 \times 480$ phase control points - more than 200 times greater than the largest conventional DM). Figure 2 shows the Hamamatsu LC SLM assembly.

One disadvantage of the current LC SLM technology is the limitation in the amplitude of the phase control $(\sim 1$ wave). This requires the use of phase wrapping techniques in order to compensate for aberrations commonly encountered in the clinical population. The use of phase wrapping techniques, in turn, constrains the range of optical wavelengths over which the wave aberration correction is valid. Visual performance testing is, therefore, restricted to monochromatic scenes, which limits the 
clinical utility. Nevertheless, the high resolution capability of this system makes it attractive for some types of psychophysical testing.
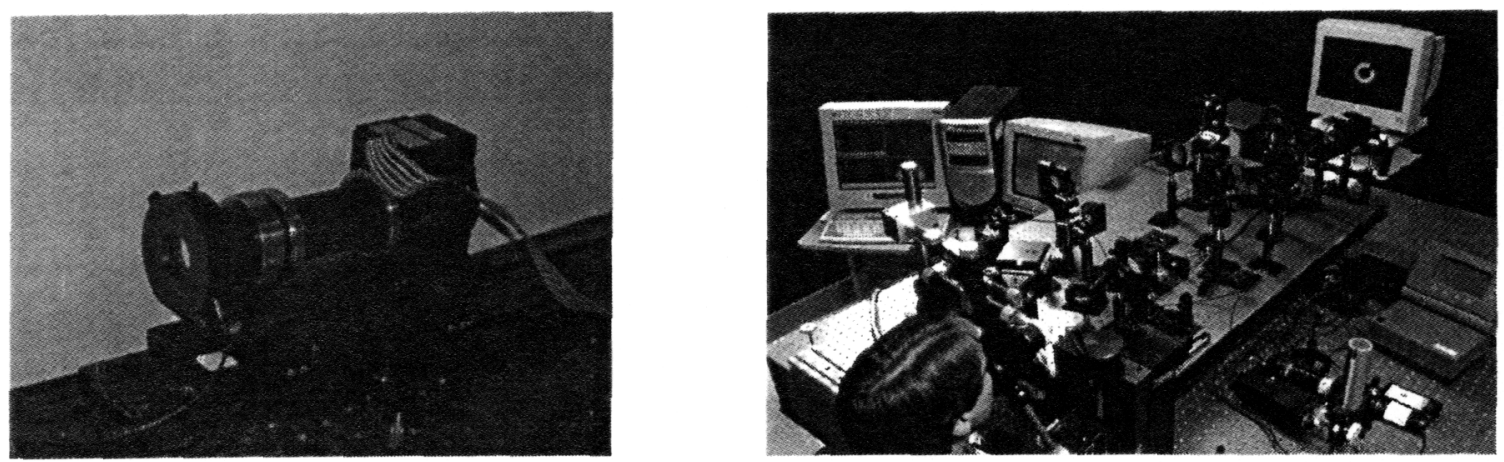

Figure 2 - Liquid crystal spatial light modulator assembly from Hamamatsu (left). Complete adaptive phoropter system using LC SLM at UC Davis Medical Center (right).

\subsection{LC SLM Adaptive Phoropter}

Using the high-resolution LC SLM technology, an adaptive phoropter has been developed at LLNL for use at the UC Davis Medical Center. The clinical goals of this system include determining the ultimate limits of visual acuity and studying the relationship between normal aging, retinal disease and visual performance through psycho-physical testing. Figure 2 also shows a complete view of the system being operated at UC Davis.

\subsection{Test results}

The LC SLM AO system has been tested with an externally induced aberration created by a lens and using an optomechanical surrogate eye assembly in place of a human eye. In this configuration, the system converges with high accuracy, resulting in a final residual error of $\sim 50 \mathrm{~nm} \mathrm{rms}$, as shown in Figure 3. Initial testing with human subjects is underway.
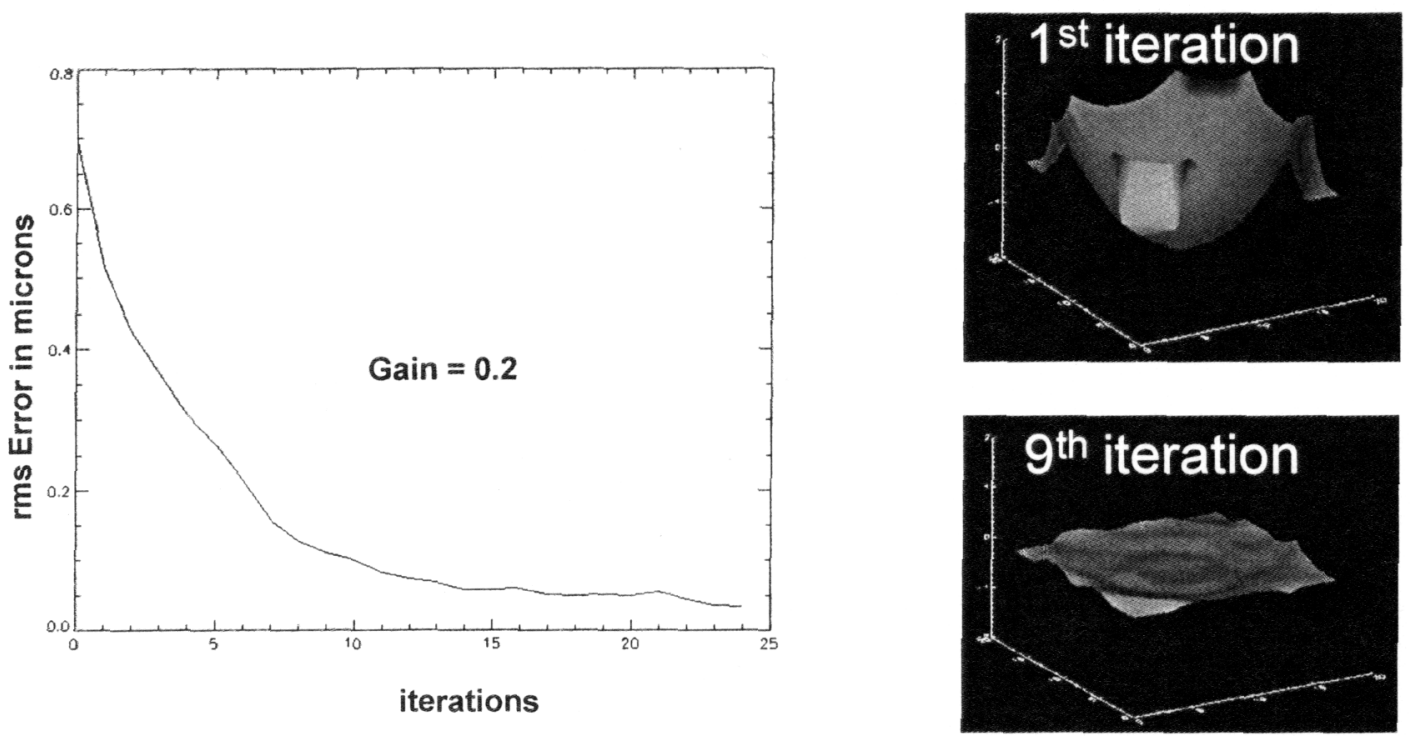

Figure 3 - Convergence of the LC SLM AO system on a defocus aberration introduced using a simple lens and an optomechanical surrogate eye. The system reaches a steady state with a residual rms error of $\sim 50 \mathrm{~nm}$ after $\sim 15$ iterations with a loop gain of 0.2 . 


\section{MEMS DEFORMABLE MIRROR VISION SYSTEM}

\subsection{MEMS DM Technology}

MEMS DM devices are commercially available from Boston Micromachines, and have been previously demonstrated at the University of Rochester in an adaptive optics laboratory test-bed to correct the wave aberrations of the human eye [10]. Figure 4 shows the MEMS DM assembly from Boston Micromachines. Advantages of the MEMS DM include compactness (clear aperture of $3 \mathrm{~mm})$, low cost $(\sim 25 \mathrm{k}$ ), ease of use (driven with standard PC frame grabber card), and good resolution (12x12 actuators - comparable to conventional DM technology used for high-end laboratory vision AO systems).

One disadvantage of the current MEMS DM technology is the limited range of surface deformation ( 1-2 microns). This limitation restricts the fraction of the clinical population over which a good wave aberration correction can be achieved. Ongoing work, sponsored by the CfAO, is proceeding to develop a new generation of high-stroke MEMS DM devices, with actuator ranges of 4-12 microns, depending on the details of the different designs under development by several groups around the country.
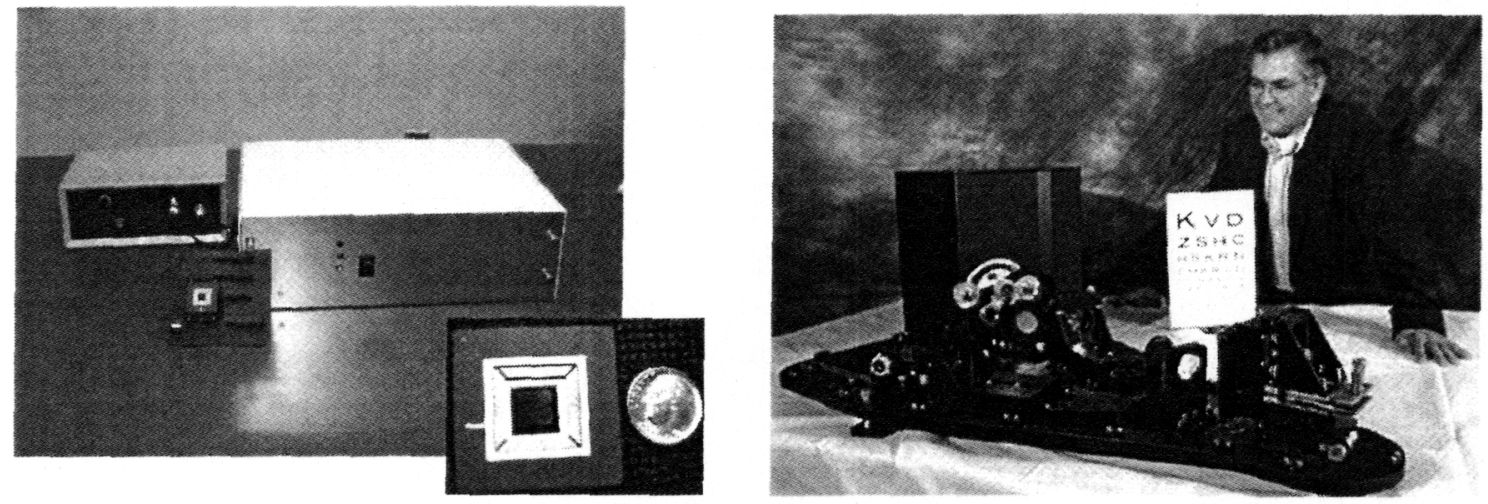

Figure 4 - MEMS deformable mirror system from Boston Micromachines (left). Complete assembled portable MEMS-based adaptive phoropter at LLNL.

\subsection{MEMS DM Adaptive Phoropter}

Using the MEMS DM technology from Boston Micromachines, along with commercially available ophthalmic wavefront sensing system from Wavefront Sciences, a portable adaptive phoropter has been developed at LLNL for initial use at the University of Rochester. The initial clinical goal for this system is to achieve comparable performance for human subjects to the laboratory adaptive optics system at the University of Rochester, in a direct, side-by-side comparison. Once this level of performance is verified, the system will be transferred to the vision clinic at Bausch \& Lomb in order to assess the visual performance enhancement due to the correction of high-order wave aberrations for a larger clinical population. Figure 4 also shows the fully assembled system at LLNL, prior to its shipment to Rochester.

\subsection{Test results}

The MEMS DM adaptive phoropter has undergone testing at LLNL using an opto-mechanical surrogate eye in place of a human eye. A lens has been used to introduce a simple defocus error. Figure 5 shows the results of a test using a 0.25 diopter lens. After closing the adaptive optics loop, the system converges to remove the defocus to a level of 0.01 diopter. The results shown in Figure 5 contain a small amount of residual astigmatism at the level of 0.13 diopters. Subsequent testing has indicated that this error is due primarily to a difference in calibration between the closed-loop system and the wavefront analysis mode used to assess the performance. A simple calibration procedure should eliminate this error source in future tests. 

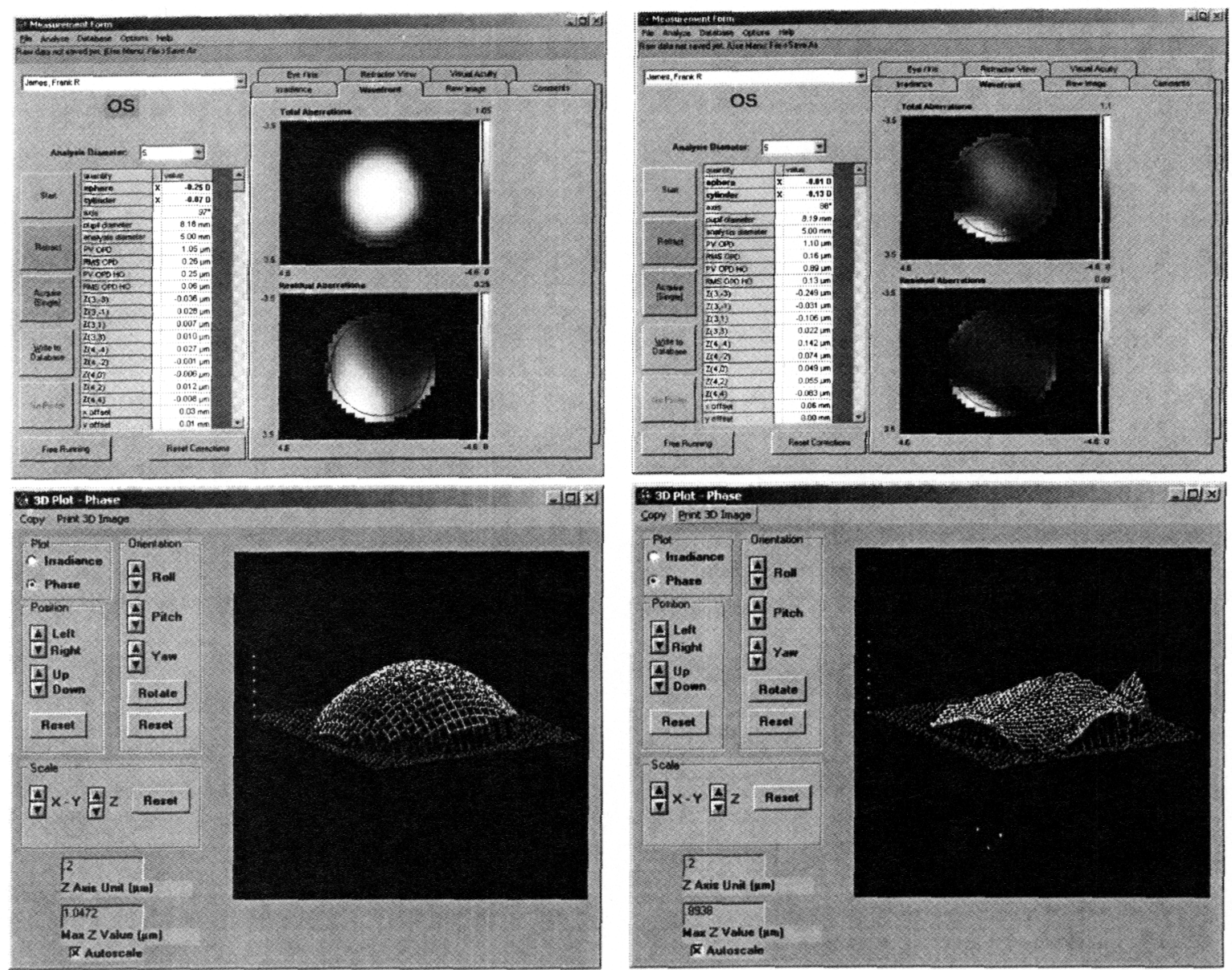

Figure 5 - Wavefront analysis for an aberration introduced using a 0.25 diopter lens and an opto-mechanical surrogate eye before closing the adaptive optics loop (left) and after closing the loop (right) using the MEMS DM. The analysis system accurately measures the defocus caused by the lens, and the closed loop system accurately removes the defocus to a level of 0.01 diopters. A residual astigmatism of $\sim 0.1$ diopter is present in the system, primarily due to a simple calibration error. Further work will eliminate this error source.

\section{SUMMARY AND CONCLUSIONS}

Two adaptive phoropter systems using novel, compact, inexpensive wavefront control technology have recently been developed and tested. The purpose of these systems is to perform both measurement and correction of the eye's aberrations up to high spatial frequencies, thereby allowing subjects to make a direct assessment of the subjective benefit of high-order wavefront correction. One of these adaptive phoropter systems uses a custom Shack-Hartmann wavefront sensor and a commercially available optically addressed LC SLM to perform high-order wavefront correction. The second adaptive phoropter system uses a commercially available Shack-Hartmann wavefront sensor and a commercially available MEMS DM for wavefront correction. The LC SLM based system has been deployed at the University of California, Davis Medical Center and is being evaluated using human subjects. The MEMS based system, which has been designed in a portable, modular configuration for clinical use, is being deployed at the University of Rochester for initial testing using human subjects and will be compared directly with the laboratory adaptive optics system at Rochester using conventional deformable mirror technology. The new wavefront corrector technologies employed have the potential to enable the development of clinical adaptive phoropters, due to their small size and low cost compared with conventional deformable mirrors. 


\section{ACKNOWLEDGEMENTS}

This work was performed under the auspices of the U.S. Department of Energy by University of California Lawrence Livermore National Laboratory under contract No. W-7405-Eng-48. This work was primarily supported by the DOE Biomedical Engineering Program. Additional support for this work was provided by the National Science Foundation Center for Adaptive Optics and by Bausch \& Lomb. Initial stages of this work were supported by the LLNL LDRD Program. This paper presents an overview of work performed by many researchers at LLNL including A. Awwal, B. Bauman, D. Gavel, S. Jones, K. O'Brien, R. Sawvel, D. Silva, C. Thompson, J. Tucker, \& S. Wilks, in collaboration with researchers at Sandia National Laboratory (S. Eisenbies, S. Haney), University of California, Davis (T. Barnes, J. Hardy, J. Werner), University of Rochester (N. Doble, D. Williams), University of Southern California (M. Humayan, S. Sadda), University of California, Berkeley (J. Flannery), US Army Aeromedical Research Lab (C. VanDePol), Bausch \& Lomb (I. Cox), Wavefront Sciences (D. Neal), Boston Micromachines (P. Bierden) and Hamamatsu (M. Wu).

\section{REFERENCES}

1. R. Zacharias, et al, "Wavefront control of high-power laser beams in the National Ignition Facility (NIF)", Proc. SPIE 3889, 332-343, 2000.

2. C. E. Max et al, "Image improvement from a sodium-layer laser guide star adaptive optics system", Science 277, 1649$1652,1997$.

3. D. T. Gavel et al, "Science with laser guide stars at Lick Observatory", Proc. SPIE 4494, 336-342, 2002.

4. P. Wizinowich et al, "First light adaptive optics images from the Keck II Telescope: a new era of high angular resolution imagery", Publications of the Astronomical Society of the Pacific 112, 315-319, 2000.

5. A. R. Contos et al, "Laser guide star adaptive optics at the Keck Observatory" Proc. SPIE 4839, p. 370-380, 2003.

6. S. S. Olivier, "Advanced adaptive optics technology development," Proc.SPIE, 4494, 1-10, 2002.

7. S. S. Olivier et al, "Micro-electro-mechanical systems spatial light modulator development" Proc. SPIE 4124, 26-31, 2000.

8. S. C. Wilks et al, "High-resolution adaptive optics test-bed for vision science," Proc. SPIE 4494, 349-356, 2002.

9. J. Liang et al, "Supernormal vision and high-resolution retinal imaging through adaptive optics," Journal of the Optical Society of America A, 14, 2884-2892, 1997.

10. N. Doble et al, "Use of a microelectromechanical mirror for adaptive optics in the human eye," Optics Letters, 27, $1537-$ $1539,2002$. 